\title{
Catálogo dos “Turbellaria” (Platyhelminthes) do Estado de São Paulo
}

\author{
Eudóxia Maria Froehlich ${ }^{1,3}$ \& Fernando Carbayo ${ }^{2}$ \\ ${ }^{1}$ Departamento de Zoologia, Instituto de Biociências, Universidade de São Paulo - USP, Rua do Matão, \\ Trav. 14, 321, Cidade Universitária, CEP 05508-900, São Paulo, SP, Brasil \\ ${ }^{2}$ Escola de Artes, Ciências e Humanidades, Universidade de São Paulo - USP, Av. Arlindo Bettio, 1000, \\ CEP 03828-000, São Paulo, SP, Brasil, e-mail: baz@usp.br \\ ${ }^{3}$ Autor para correspondência: Eudóxia Maria Froehlich,e-mail: emfroeh@ib.usp.br
}

FROEHLICH, E.M. \& CARBAYO, F. Checklist of "Turbellaria” (Platyhelminthes) from São Paulo State, Brazil. Biota Neotrop. 11(1a): http://www.biotaneotropica.org.br/v11n1a/en/abstract?inventory+bn0221101a2011

Abstract: A recent survey of the literature and databases on turbellarian fauna from the State of São Paulo, Brazil, yielded a total of 312 species (including Acoelomorpha, a new phylum) inhabiting marine, as well as freshwater, and terrestrial habitats. In 1999, approximately 333 species were registered. This higher number is related to a number of species synonymized thereafter, and probably related to lower accuracy of prior accounts. Nonetheless, the only two taxonomists studying this animal group in the State estimated a much higher actual number of species. In the State there are three scientific collections containing turbellarians, almost exclusively from terrestrial habitats.

Keywords: Turbellaria, biodiversity of the State of São Paulo, BIOTA/FAPESP Program.

Number of species: In the world: ca. 6500 (Schockaert et al., 2008), in Brazil: 467 (Carbayo et al., 2009), estimated in São Paulo state: 312.

FROEHLICH, E.M. \& CARBAYO, F. Catálogo dos “Turbellaria" (Platyhelminthes) do Estado de São Paulo, Brasil. Biota Neotrop. 11(1a): http://www.biotaneotropica.org.br/v11n1a/pt/abstract?inventory+bn0221101a2011

Resumo: Mediante consulta bibliográfica e de bases de dados encontramos registros para o estado de São Paulo de 312 espécies de turbelários (incluindo Acoelomorpha, novo filo) de ambientes marinhos, límnicos e terrestres. Em 1999 haviam sido registradas aproximadamente 333 espécies; o número maior deve ser atribuído a espécies posteriormente sinonimizadas e, provavelmente, à precisão menor dos cômputos anteriores. Os dois únicos pesquisadores especialistas do grupo, trabalhando no estado, estimam, porém, um número muito maior. Há três coleções científicas abrigando turbelários, quase exclusivamente, terrestres.

Palavras-chave: Turbellaria, biota paulista, Programa BIOTA/FAPESP.

Número de espécies: No mundo: ca. 6500 (Schockaert et al., 2008), no Brasil: 467 (Carbayo et al., 2009), estimadas no estado de São Paulo: 312. 


\section{Introdução}

Os turbelários, vermes do filo Platyhelminthes, na sua grande maioria de vida livre, são animais de simetria bilateral, acelomados. Possuem epiderme ciliada e tubo digestivo com fundo cego, desprovido de ânus. São hermafroditas e, caracteristicamente, de hábitos predadores. Vivem em ambientes aquáticos - marinhos ou de água doce, ou em ambientes terrestres úmidos. Em geral muito pequenos, a maioria tem de um a poucos milímetros, mas em dois grupos, um marinho e outro terrestre, com comprimento da ordem de centímetros, há formas que podem atingir meio metro ou mais.

É conhecida a natureza parafilética dos "Turbellaria", classe taxonômica atualmente constituída por 10 ordens: Catenulida, Haplopharyngida, Lecithoepitheliata, Macrostomida, Polycladida, Prolecithophora, Proseriata, Rhabdocoela, Temnocephalida e Tricladida (Cannon 1986, Rieger et al. 1991). As relações de parentesco entre os grupos são ainda mal compreendidas, e a monofilia de algumas ordens é matéria de discussão (Noren \& Jondelius 2002).

Até recentemente as ordens Acoela e Nemertodermatida faziam parte dos "Turbellaria". Hoje constituem o novo filo Acoelomorpha Baguñà \& Riutort 2004, de natureza parafilética. Neste trabalho, porém, em que nos referimos aos turbelários, incluímos, por conveniência, as espécies de Acoelomorpha.

\section{Metodologia}

A lista de espécies de turbelários registrados para o Estado de São Paulo foi elaborada mediante consultas no nosso acervo bibliográfico e mediante uma primeira busca nas bases de dados Biological Abstracts e Zoological Record (Thomson Reuters) com as palavras-chave Brazil e Turbellaria, e/ou Polycladida, e/ ou Tricladida (agosto 2008) e uma segunda busca com as palavras chave Brazil e Turbellaria (maio 2010). Das referências obtidas foram selecionadas as que registram estudos ou ocorrências de turbelários para o Estado de São Paulo. Os nomes das espécies foram atualizados segundo Tyler et al. (2006-2009). Uma lista de referências de macroturbelários de todo o Brasil pode ser consultada em Carbayo \& Froehlich (2008).

\section{Resultados e Discussão}

Para o estado de São Paulo há registros de 176 espécies marinhas, 83 límnicas e 53 terrestres, compondo um total de 312 espécies de

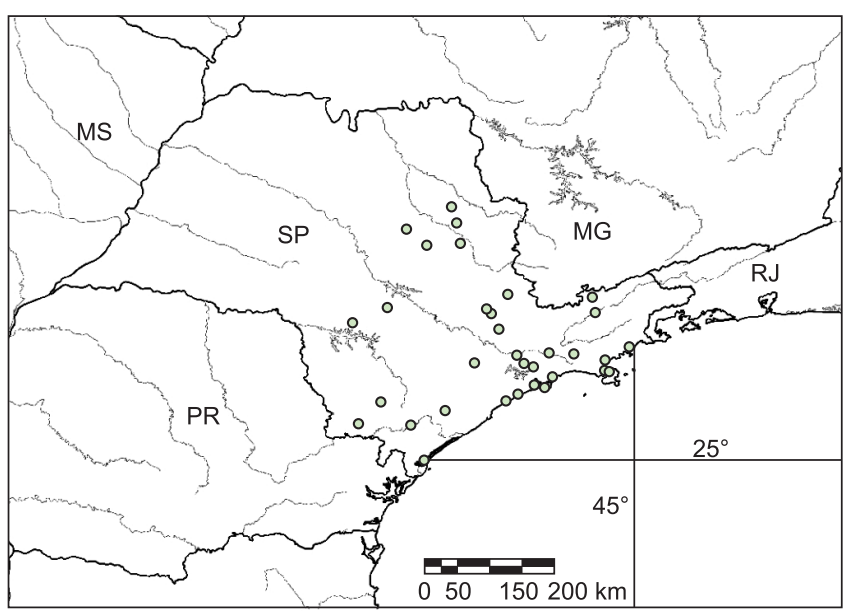

Figura 1. Localidades com registro de turbelários para o estado de São Paulo. turbelários (Tabela 1) registradas em 37 municípios (Figura 1). Este número de espécies representa dois terços das espécies conhecidas para o Brasil, o que é muito mais consequência do esforço maior de coleta no estado, do que da riqueza real estimada de espécies para o país. A falta de registros de turbelários na metade ocidental do estado (Figura 1) deve-se ao fato de a área nunca ter sido prospectada com esta finalidade.

Na série de publicações Biodiversidade do estado de São Paulo, organizada por Joly \& Bicudo (1999), Forneris (1999) e Rodrigues \& Froehlich (1998) citaram para o estado de São Paulo, 200 espécies, aproximadamente, e 81 espécies, respectivamente, de turbelários de ambientes marinhos e turbelários de ambientes límnicos. Naquela ocasião não foi feito um levantamento da fauna de ambientes terrestres, constando de 52 espécies, o que elevaria para 333 o número total de espécies nominais conhecidas para o estado. Após 1999 houve apenas mais um registro de espécie terrestre nativa, Notogynaphallia ernesti Leal-Zanchet \& Froehlich, 2003, além de uma espécie introduzida, e mais 12 espécies do filo Acoelomorpha (Hooge \& Rocha 2006). Posteriormente, Carbayo et al. (2009) contabilizaram 317 espécies para o estado. Estas diferenças numéricas nos censos se devem à imprecisão (as 200 espécies citadas por Rodrigues \& Froehlich (1998) eram uma aproximação) e à sinonimização de várias espécies nominais.

Existem no estado dois taxonomistas especialistas em turbelários, os autores, ambos estudiosos da fauna de planárias terrestres. Em outros estados do Brasil há mais três especialistas: Ana Maria Leal Zanchet (Unisinos), que estuda planárias terrestres, além de microturbelários límnicos, e Suzana B. Amato (UFRGS) e José F. R. Amato (UFRGS), que estudam temnocefalídeos, turbelários límnicos epibiontes.

No estado de São Paulo existem três coleções de turbelários. A mais antiga delas, sob os cuidados do primeiro autor, acolhe quase todos os tipos, não nomeados formalmente, das espécies brasileiras descritas por Marcus, du Bois-Reymond Marcus, C. G. Froehlich e E. M. Froehlich. O segundo autor mantém uma coleção com ca. 4500 espécimes de planárias terrestres, principalmente da mata Atlântica dos estados de ES, RJ, SP, PR, SC e RS. No Museu de Zoologia da USP estão depositados aproximadamente 800 espécimes, quase todos de planárias terrestres. Desses, 48 espécimes são tipos, principalmente de Acoelomorpha. A Profa. Dra. Suzana B. Amato mantém na UFRGS um número expressivo de espécimes pertencentes a dez espécies conhecidas de Temnocephala (Temnocephalida) dos estados de PA, MT, MG, SP, PR, SC e RS, além de espécimes de seis espécies novas para a ciência que estão em processo de descrição. Na Unisinos (São Leopoldo, RS) existem vários milhares de espécimes de Tricladida.

A fauna conhecida de turbelários do estado é uma pequena fração da real, mas é difícil estimar com alguma precisão a diversidade real. Sobre a distribuição geográfica das espécies só se tem registro, na maioria dos casos a localidade tipo. A afirmação de que alguns táxons de turbelários apresentam elevado grau de endemismo (Sluys 1999, Carbayo et al. 2002) deve ser acolhida com precaução em face das relativamente poucas regiões amostradas (Rodrigues \& Froehlich 1998). À medida que as coletas se estenderem a outras regiões, novos registros para espécies conhecidas, além de novas espécies, poderão ser acrescentados. Assim foi constatado para as planárias terrestres, único grupo taxonômico de turbelários que vem recebendo atenção no estado. Mesmo nas áreas mais estudadas, como o município de São Paulo e arredores, têm sido encontradas, recentemente, espécies de planárias terrestres novas para a ciência. Os autores possuem um grande número de espécies de planárias terrestres não descritas, à espera de novos taxonomistas para estudá-las e catalogá-las. 
Tabela 1. Espécies de "Turbellaria" + Acoelomorpha registradas para o estado de São Paulo e município onde foram registradas.

Table 1. "Turbellaria"+ Acoelomorpha species recorded for São Paulo State with indication of municipalities where they were recorded.

\begin{tabular}{|c|c|c|c|}
\hline $\mathbf{N}^{\circ}$ & Táxon & Espécie descritor & $\begin{array}{c}\text { Ocorrência } \\
\text { [em município(s) do estado SP] }\end{array}$ \\
\hline 1 & Acoelomorpha & Amphiscolops evelinae Marcus, 1947 & Ubatuba, Santos \\
\hline 2 & & Archaphanostoma marcusi Hooge \& Rocha, 2006 & Ilhabela \\
\hline 3 & & Avagina marci Dörjes and Karling, 1975 & São Sebastião, Ilhabela \\
\hline 4 & & Childia groenlandica (Levinsen, 1879) & Santos \\
\hline 5 & & Childia westbladi (Marcus, 1950) & Ilhabela, Santos \\
\hline 6 & & Convoluta hensensi Bohmig, 1895 & São Sebastião, Cananéia \\
\hline 7 & & Deuterogonaira thauma (Marcus, 1952) & Ilhabela, Santos \\
\hline 8 & & Eumecynostomum evelinae Marcus, 1948 & São Sebastião, Guarujá, São Vicente \\
\hline 9 & & Faerlea antora Marcus, 1951 & Ilhabela \\
\hline 10 & & Hallangia evelinae Marcus, 1952 & Ilhabela \\
\hline 11 & & Haplocelis dichona (Marcus, 1954) & São Sebastião \\
\hline 12 & & Haplogonaria pellita (Marcus, 1951) & São Sebastião \\
\hline 13 & & Haplogonaria sophiae Hooge \& Rocha, 2006 & São Sebastião \\
\hline 14 & & Haploposthia microphoca Marcus, 1950 & Ilhabela \\
\hline 15 & & Heterochaerus carvalhoi (Marcus, 1952) & Bertioga, Santos, Cananéia \\
\hline 16 & & Heterochaerus sargassi (Hyman, 1939) & São Sebastião, Ilhabela \\
\hline 17 & & Isodiametra divae (Marcus, 1950) & São Sebastião, Ilhabela \\
\hline 18 & & Isodiametra vexillaria (Marcus, 1948) & São Sebastião, Santos \\
\hline 19 & & Isodiametra westbladi (Macus, 1949) & Ilhabela, Ubatuba, Caraguatatuba \\
\hline 20 & & Kuma albiventer (Marcus, 1954) & Ilhabela \\
\hline 21 & & Kuma asilhas Hooge \& Rocha, 2006 & São Sebastião \\
\hline 22 & & Kuma belca Marcus, 1952 & Ubatuba \\
\hline 23 & & Kuma brevicauda Marcus, 1950 & Cananéia \\
\hline 24 & & Mecynostomum tenuissimum (Westblad, 1946) & São Sebastião, Ilhabela \\
\hline 25 & & Otocelis erinae Hooge \& Rocha, 2006 & São Sebastião \\
\hline 26 & & Paraproporus xanthus Marcus, 1950 & Ilhabela \\
\hline 27 & & Paratomella rubra Rieger \& Ott, 1971 & São Sebastião \\
\hline 28 & & Philactinoposthia coneyi Hooge \& Rocha, 2006 & Ilhabela \\
\hline 29 & & Philactinoposthia stylifera brasiliensis Hooge \& Rocha, 2006 & São Sebastião \\
\hline 30 & & Philocelis robrochai Hooge \& Rocha, 2006 & Ilhabela \\
\hline 31 & & Pseudanaperus tinctus (Marcus, 1952) & Ilhabela \\
\hline 32 & & Pseudaphanostoma divae Marcus, 1952 & São Sebastião, Ilhabela, Ubatuba \\
\hline 33 & & Pseudaphanostoma herringi Hooge \& Rocha, 2006 & Ilhabela \\
\hline 34 & & Pseudokuma orphinum (Marcus, 1950) & Ilhabela \\
\hline 35 & Catenulida & Anokkostenostomum anatirostrum (Marcus, 1945) & São Paulo \\
\hline 36 & & Anokkostenostomum corderoi (Marcus, 1945) & São Paulo \\
\hline 37 & & Anokkostenostomum membranosum (Kepner \& Carter, 1931) & São Paulo \\
\hline 38 & & Anokkostenostomum pegephilum (Nuttycombe \& Waters, 1938) & São Paulo \\
\hline 39 & & Anokkostenostomum pseudoacetablum (Nuttycombe \& Waters, 1935) & São Paulo \\
\hline 40 & & Anokkostenostomum saliens (Kepner \& Carter, 1931) & São Paulo \\
\hline 41 & & Anokkostenostomum tuberculosum (Nuttycombe \& Waters, 1938) & Campinas, São Paulo \\
\hline 42 & & Anokkostenostomum ventronephrium Nuttycombe, (1932) & São Paulo \\
\hline 43 & & Anokkstenostomum evelinae (Marcus, 1945) & São Paulo, Santa Rita de Passa Quatro \\
\hline 44 & & Catenula alitha Marcus, 1945 & São Paulo \\
\hline 45 & & Catenula lemnae Duges, 1832 & São Paulo, Santa Rita de Passa Quatro \\
\hline 46 & & Catenula leuca Marcus, 1945 & São Paulo \\
\hline 47 & & Catenula macrura Marcus, 1945 & São Paulo \\
\hline 48 & & Chordarium cryptum Marcus, 1945 & São Paulo \\
\hline
\end{tabular}


Tabela 1. Continuação...

\begin{tabular}{|c|c|c|c|}
\hline $\mathbf{N}^{\circ}$. & Táxon & Espécie descritor & $\begin{array}{c}\text { Ocorrência } \\
\text { [em município(s) do estado SP] }\end{array}$ \\
\hline 49 & Catenulida & Chordarium evelinae Marcus, 1945 & São Paulo \\
\hline 50 & & Chordarium leucanthum Marcus, 1945 & $\begin{array}{l}\text { Santa Rita de Passa Quatro, } \\
\text { Campinas, São Paulo }\end{array}$ \\
\hline 51 & & Chordarium philum Marcus, 1945 & São Paulo \\
\hline 52 & & Dasyhormus lasius Marcus, 1945 & São Paulo \\
\hline 53 & & Dasyhormus lithophorus Marcus, 1945 & São Paulo \\
\hline 54 & & Rhynchoscolex evelinae Marcus, 1945 & São Paulo \\
\hline 55 & & Rhynchoscolex nanus Marcus, 1945 & São Paulo, Santa Rita de Passa Quatro \\
\hline 56 & & Rhynchoscolex platypus Marcus, 1945 & São Paulo \\
\hline 57 & & Rhynchoscolex pusillus Marcus, 1945 & São Paulo \\
\hline 58 & & Rhynchoscolex simplex Leidy, 1851 & São Paulo \\
\hline 59 & & Stenostomum amphotum Marcus, 1945 & Campinas, São Paulo \\
\hline 60 & & Stenostomum arevaloi Gieysztor, 1931 & Campinas, São Paulo \\
\hline 61 & & Stenostomum bicaudatum Kennel, 1888 & São Paulo \\
\hline 62 & & Stenostomum ciliatum Kepner \& Carter, 1931 & São Paulo \\
\hline 63 & & Stenostomum cryptops Nuttycombe \& Waters, 1935 & São Paulo \\
\hline 64 & & Stenostomum glandulosum Kepner \& Carter, 1931 & São Paulo \\
\hline 65 & & Stenostomum grande Child, 1902 & São Paulo \\
\hline 66 & & Stenostomum hemisphericum Nasonov, 1924 & São Paulo \\
\hline 67 & & Stenostomum leucops (Duges, 1828) & São Paulo \\
\hline 68 & & Stenostomum matarazzoi Marcus, 1949 & São Paulo \\
\hline 69 & & Stenostomum paraguayense (Martin, 1908) & Campinas, São Paulo \\
\hline 70 & & Stenostomum rosulatum Marcus, 1945 & São Paulo \\
\hline 71 & & Stenostomum simplex Kepner \& Carter, 1931 & São Paulo \\
\hline 72 & & Stenostomum uronephrium Nuttycombe, 1931 & São Paulo \\
\hline 73 & & Stenostomum virginianum Nuttycombe, 1931 & $\begin{array}{c}\text { Campinas, } \\
\text { Santa Rita do Passa Quatro, São Paulo }\end{array}$ \\
\hline 74 & & Suomina evelinae Marcus, 1945 & São Paulo \\
\hline 75 & & Suomina sawayai Marcus, 1945 & São Paulo \\
\hline 76 & & Suomina turgida (Zacharias, 1902) & São Paulo \\
\hline 77 & Kalyptorhynchia & Alcha evelinae Marcus, 1949 & Santos \\
\hline 78 & & Gyratrix hermaphroditus Ehrenberg, 1831 & São Paulo \\
\hline 79 & & Harsa obnixa Marcus, 1951 & São Sebastião, Caraguatatuba, Cananéia \\
\hline 80 & & Oneppus timius Marcus, 1952 & São Sebastião, Caraguatatuba \\
\hline 81 & & Rhinolasius sartus Macus, 1951 & Caraguatatuba, Cananéia \\
\hline 82 & & Toia ycia Marcus, 1952 & Ilhabela, Santos \\
\hline 83 & Macrostomida & Archimacrostomum beaufortense (Ferguson, 1937) & Santos, São Vicente \\
\hline 84 & & Archimacrostomum brasiliensis (Marcus, 1952) & São Sebastião, Caraguatatuba \\
\hline 85 & & Dolichomacrostomum mortenseni Marcus, 1950 & Ilhabela \\
\hline 86 & & Karkingia lutheri (Marcus, 1948) & Guarujá, Santos \\
\hline 87 & & Macrostomum delphax Marcus, 1946 & São Paulo \\
\hline 88 & & Macrostomum evelinae Marcus, 1946 & Ilhabela, Guarujá \\
\hline 89 & & Microstomum breviceps Marcus, 1951 & Ilhabela \\
\hline 90 & & Microstomum gabriellae Marcus, 1950 & Ilhabela, Ubatuba \\
\hline 91 & & Microstomum rhabdotum Marcus, 1951 & Ilhabela \\
\hline 92 & & Microstomum trichotum Marcus, 1950 & Ilhabela \\
\hline 93 & & Microstomum ulum Marcus, 1950 & São Sebastião, Ilhabela \\
\hline 94 & & Myozona evelinae Marcus, 1949 & São Sebastião, Ilhabela, Ubatuba \\
\hline 95 & Polycladida & Acerotisa bituna Marcus, 1947 & Guarujá \\
\hline
\end{tabular}


Tabela 1. Continuação...

\begin{tabular}{|c|c|c|c|}
\hline $\mathbf{N}^{\circ}$ & Táxon & Espécie descritor & $\begin{array}{c}\text { Ocorrência } \\
\text { [em município(s) do estado SP] }\end{array}$ \\
\hline 96 & Polycladida & Acerotisa leuca Marcus, 1947 & Guarujá \\
\hline 97 & & Adenoplana evelinae Marcus, 1950 & Ilhabela \\
\hline 98 & & Alloioplana aulica (Marcus, 1947) & Guarujá \\
\hline 99 & & Armatoplana leptalea (Marcus, 1947) & Guarujá \\
\hline 100 & & Callioplana evelinae Marcus, 1954 & Ilhabela \\
\hline 101 & & Cestoplana salar Marcus, 1949 & Ilhabela \\
\hline 102 & & Cestoplana techa du-Bois-Reymond Marcus, 1957. & Ilhabela \\
\hline 103 & & Comoplana angusta (Verrill, 1893) & Guarujá, São Vicente \\
\hline 104 & & Cycloporus gabriellae Marcus, 1950 & Ilhabela, Ubatuba \\
\hline 105 & & Distylochus isifer (du Bois Reymond Marcus, 1955) & Cananeia \\
\hline 106 & & Distylochus martae (Marcus, 1947) & Guarujá \\
\hline 107 & & Duplominona tridens (Marcus, 1954) & São Sebastião, Ilhabela \\
\hline 108 & & Enchiridium evelinae Marcus, 1949 & São Sebastião, Santos \\
\hline 109 & & Euplana hymanae Marcus, 1947 & Santos \\
\hline 110 & & Euprosthiostomum mortensenii Marcus, 1948 & São Vicente \\
\hline 111 & & Euprothiostomum matarazzoi Marcus, 1950 & Ilhabela \\
\hline 112 & & Eurylepta neptis Du-Bois Reymond Marcus, 1955 & Ilhabela \\
\hline 113 & & Eurylepta piscatoria (Marcus, 1947) & Guarujá \\
\hline 114 & & Eurylepta turma Marcus, 1952 & Ilhabela, Ubatuba \\
\hline 115 & & Hoploplana divae Marcus, 1950 & Ilhabela \\
\hline 116 & & Hoploplana usaguia Smith, 1960 & Ubatuba \\
\hline 117 & & Interplana evelinae (Marcus, 1952) & Guarujá, Itanhaém \\
\hline 118 & & Itannia ornata Marcus, 1947 & Ihabela, Ubatuba, Guarujá \\
\hline 119 & & Latocestus callizonica (Marcus, 1947) & Guarujá, Santos \\
\hline 120 & & Lurymare gabriellae (Marcus, 1949) & Ilhabela \\
\hline 121 & & Notocomplana evelinae (Marcus, 1947) & Guarujá \\
\hline 122 & & Notocomplana syntoma (Marcus, 1947) & São Vicente \\
\hline 123 & & Notoplana divae Marcus, 1948 & Santos \\
\hline 124 & & Notoplana martae Marcus, 1948 & Ilhabela, Santos \\
\hline 125 & & Notoplana megala Marcus, 1952 & Ilhabela, Ubatuba \\
\hline 126 & & Notoplana plecta Marcus, 1947 & Santos \\
\hline 127 & & Notoplana sawayai Marcus, 1947 & Santos \\
\hline 128 & & Notoplana syntoma Marcus, 1947 & Santos, São Vicente \\
\hline 129 & & Pentaplana divae Marcus, 1949 & São Vicente \\
\hline 130 & & Phaenocelis medvenica Marcus, 1952 & Ilhabela \\
\hline 131 & & Prolatocestus ocellatus (Marcus, 1947) & Guarujá \\
\hline 132 & & Prosthiostomum cynarium Marcus, 1950 & Ilhabela \\
\hline 133 & & Prosthiostomum gilvum Marcus, 1950 & Ilhabela \\
\hline 134 & & Prothiostomum utarum Marcus, 1952 & Ilhabela \\
\hline 135 & & Pseudobiceros evelinae (Marcus, 1950) & Santos \\
\hline 136 & & Pseudoceros chloreus Marcus, 1949 & Ilhabela \\
\hline 137 & & Pseudoceros hispidus du-Bois Reymond Marcus, 1955 & Ilhabela \\
\hline 138 & & Pseudoceros mopsus Marcus, 1952 & Ilhabela \\
\hline 139 & & Stylochoplana divae (Marcus, 1949) & Guarujá \\
\hline 140 & & Stylochoplana leptalea Marcus, 1947 & Santos \\
\hline 141 & & Stylochoplana selenopsis Marcus, 1947 & Santos, São Vicente \\
\hline 142 & & Stylochus catus du-Bois Reymond Marcus, 1958 & Ilhabela \\
\hline 143 & & Stylochus ticus Marcus, 1952 & Ilhabela \\
\hline 144 & & Theama evelinae Marcus, 1949 & Ilhabela \\
\hline
\end{tabular}


Tabela 1. Continuação...

\begin{tabular}{|c|c|c|c|}
\hline $\mathbf{N}^{\circ}$ & Táxon & Espécie descritor & $\begin{array}{c}\text { Ocorrência } \\
{[\mathrm{em} \text { município(s) do estado SP] }}\end{array}$ \\
\hline 145 & Polycladida & Thysanozoon brocchii (Risso, 1818) & Ilhabela \\
\hline 146 & & Triadomma curvum Marcus, 1949 & Ilhabela \\
\hline 147 & & Triadomma evelinae Marcus, 1947 & Guarujá \\
\hline 148 & & Zygantroplana henriettae Corrêa, 1949 & Ilhabela \\
\hline 149 & Prolecitophora & Acmostomum canarium Marcus, 1947 & Santos \\
\hline 150 & & Acmostomum evelinae (Marcus, 1951) & São Sebastião \\
\hline 151 & & Cylindrostoma hyljeum Marcus, 1952 & Ilhabela \\
\hline 152 & & Cylindrostoma ibeenum Marcus, 1950 & Ilhabela \\
\hline 153 & & Cylindrostoma myfflum Marcus, 1951 & Ilhabela \\
\hline 154 & & Cylindrostoma netsicum Marcus, 1950 & Ilhabela \\
\hline 155 & & Enterostomula evelinae Marcus, 1948 & Santos \\
\hline 156 & & Haploophorum elachisterum Marcus, 1948 & Guarujá, Santos \\
\hline 157 & & Monoophorum mutsum Marcus, 1952 & Ubatuba \\
\hline 158 & & Monoophorum tigacum Marcus, 1950 & Ilhabela \\
\hline 159 & & Plagiostomum acoluthum Marcus, 1948 & Santos \\
\hline 160 & & Plagiostomum autectum Marcus, 1948 & Santos \\
\hline 161 & & Plagiostomum clusum Marcus, 1951 & Ilhabela \\
\hline 162 & & Plagiostomum girardi Westblad, 1956 & Guarujá, Santos \\
\hline 163 & & Plagiostomum kurrum Marcus, 1951 & Ilhabela \\
\hline 164 & & Plagiostomum lapinum Marcus, 1952 & Ilhabela \\
\hline 165 & & Plagiostomum nonatoi Marcus, 1948 & Santos \\
\hline 166 & & Plagiostomum sagax Marcus, 1951 & Ilhabela \\
\hline 167 & & Plagiostomum thelotrichum Marcus, 1951 & Santos \\
\hline 168 & & Plicastoma astrum Marcus, 1947 & Santos \\
\hline 169 & & Plicastoma carvalhoi Marcus, 1947 & Santos \\
\hline 170 & & Plicastoma phocae arcus, 1947 & Santos \\
\hline 171 & & Puzostoma evelinae Marcus, 1950 & Ilhabela \\
\hline 172 & & Rosmarium evelinae Marcus, 1950 & Ilhabela \\
\hline 173 & & Thallagus divae Marcus, 1951 & Ilhabela \\
\hline 174 & & Urastoma evelinae Marcus, 1951 & Ilhabela \\
\hline 175 & & Vorticeros cyrtum Marcus, 1947 & Santos \\
\hline 176 & Proseriata & Bothrioplana semperi Braun, 1881 & São Paulo \\
\hline 177 & & Duplominona mica (Marcus, 1951) & Ilhabela \\
\hline 178 & & Inaloa scalopura (Marcus, 1949) & Ilhabela, São Vicente \\
\hline 179 & & Itaspis evelinae Marcus, 1952 & Ilhabela, Ubatuba \\
\hline 180 & & Kata evelinae Marcus, 1949 & São Sebastião, Ilhabela, Ubatuba \\
\hline 181 & & Kata leroda Marcus, 1950 & Ilhabela \\
\hline 182 & & Mesoda gabriellae Marcus, 1949 & Ilhabela \\
\hline 183 & & Mesoda thelura (Marcus, 1951) & São Sebastião \\
\hline 184 & & Monocelis tabira Marcus, 1950 & Ilhabela, Santos \\
\hline 185 & & Necia sopha Marcus, 1950 & Ilhabela, Santos \\
\hline 186 & & Nematoplana asita Marcus, 1950 & São Sebastião \\
\hline 187 & & Nematoplana naia Marcus, 1949 & São Vicente \\
\hline 188 & & Parotoplana moya Marcus, 1949 & São Sebastião, Ilhabela, São Vicente \\
\hline 189 & & Peraclistus itaipus Marcus, 1950 & Santos \\
\hline 190 & & Philosyrtis eumeca Marcus, 1950 & São Sebastião, São Vicente \\
\hline 191 & & Promonotus erinaceus Marcus, 1950 & Ilhabela \\
\hline 192 & & Promonotus villacae Marcus, 1949 & São Vicente \\
\hline 193 & & Tabaota curiosa Marcus, 1950 & São Sebastião \\
\hline
\end{tabular}


Tabela 1. Continuação...

\begin{tabular}{|c|c|c|c|}
\hline $\mathbf{N}^{\circ}$ & Táxon & Espécie descritor & $\begin{array}{c}\text { Ocorrência } \\
\text { [em município(s) do estado SP] }\end{array}$ \\
\hline 194 & Proseriata & Togarma evelinae Marcus, 1949 & Ilhabela \\
\hline 195 & & Vannuccia martae Marcus, 1948 & Guarujá \\
\hline 196 & Rhabdocoela & Alcha evelinae Marcus, 1949 & São Sebastião, Santos \\
\hline 197 & & Anoplodium evelinae (Marcus, 1949) & Santos \\
\hline 198 & & Artinga evelinae Marcus, 1948 & Guarujá, Santos \\
\hline 199 & & Baicalellia evelinae Marcus, 1946 & São Paulo, Botucatu, Campos do Jordão \\
\hline 200 & & Brinkmanniella augusti Marcus, 1951 & Ilhabela \\
\hline 201 & & Byrsophlebs lutheri (Marcus, 1952) & Ilhabela, São Vicente. Cananéia \\
\hline 202 & & Chelipana (Rhinepera) targa (Marcus, 1952) & Santos, São Vicente \\
\hline 203 & & Cheliplana asica Marcus, 1952 & Ilhabela, Caraguatatuba, Cananeia \\
\hline 204 & & Daelja secuta Marcus, 1951 & Cananéia \\
\hline 205 & & Daelja secuta Marcus, 1951 & Cananéia \\
\hline 206 & & Gieysztoria (Marcusiella) thymara (Marcus, 1946) & São Paulo \\
\hline 207 & & Gieysztoria acariaia (Marcus, 1946) & São Paulo \\
\hline 208 & & Gieysztoria bellis (Marcus, 1946) & São Paulo \\
\hline 209 & & Gieysztoria cypris (Marcus, 1946) & São Paulo \\
\hline 210 & & Gieysztoria evelinae (Marcus, 1946) & São Paulo \\
\hline 211 & & Gieysztoria hymanae (Marcus, 1946) & São Paulo \\
\hline 212 & & Gieysztoria intricata (Marcus, 1946) & São Paulo \\
\hline 213 & & Gieysztoria ornata (Hofsten, 1907) & São Paulo \\
\hline 214 & & Gieysztoria therapaina (Marcus, 1946) & Campos do Jordão \\
\hline 215 & & Gieysztoria tridesma (Marcus, 1946) & Tremembé, São Paulo \\
\hline 216 & & Gieysztoria trisolena (Marcus, 1946) & São Paulo \\
\hline 217 & & Gieysztoria uncia marcus, 1946 & São Paulo \\
\hline 218 & & Haloplanella ibla Marcus, 1952 & Ilhabela \\
\hline 219 & & Itaipusa divae Marcus, 1949 & Santos, Praia Grande \\
\hline 220 & & Kalyla gabriellae Marcus, 1951 & Guarujá, São Vicente \\
\hline 221 & & Lenopharynx triops Marcus, 1951 & Ilhabela \\
\hline 222 & & Lurus evelinae Marcus, 1950 & Santos \\
\hline 223 & & Memyla phocanella Marcus, 1952 & Caragutatuba \\
\hline 224 & & Microdalyellia sawayai Marcus, 1946 & Botucatu \\
\hline 225 & & Paulodora matarazzoi Marcus, 1948 & Santos \\
\hline 226 & & Pogaina suslica (Marcus, 1951) & São Sebastião \\
\hline 227 & & Promesostoma scylax Marcus, 1952 & Ilhabela \\
\hline 228 & & Proschizorhynchus atopus Marcus, 1950 & Ilhabela \\
\hline 229 & & Ruanis pandula Marcus, 1952 & Ilhabela \\
\hline 230 & & Schizorhynchoides martae Marcus, 1950 & Cananéia \\
\hline 231 & & Trapichorhynchus tapes Marcus, 1949 & Ilhabela \\
\hline 232 & & Trigonostomum divae Marcus, 1948 & Santos, São Vicente \\
\hline 233 & & Trisaccopharynx pusa (Marcus, 1952) & Ilhabela \\
\hline 234 & & Utelga deina Marcus, 1949 & Santos \\
\hline 235 & & Zuccaria fredylina Marcus, 1948 & Santos \\
\hline 236 & & Zuccaria gabriellae Marcus, 1948 & Guarujá, Santos \\
\hline 237 & Tricladida & Bopsula evelinae Marcus, 1946 & Campos do Jordão \\
\hline 238 & & Choeradoplana iheringi von Graff, 1899 & Salesópolis, Ribeirão Pires, São Paulo \\
\hline 239 & & Choeradoplana marthae Froehlich, 1955 & Mongaguá \\
\hline 240 & & Dinizia divae Marcus, 1947 & Guarujá \\
\hline 241 & & Dolichoplana carvalhoi Corrêa, 1947 & São Paulo \\
\hline 242 & & Enterosyringa pseudorhynchodemus (Riester, 1938) & São Paulo \\
\hline
\end{tabular}


Tabela 1. Continuação...

\begin{tabular}{|c|c|c|c|}
\hline $\mathbf{N}^{\circ}$. & Táxon & Espécie descritor & $\begin{array}{c}\text { Ocorrência } \\
{[\text { [em município(s) do estado SP] }}\end{array}$ \\
\hline 243 & Tricladida & Geobia subterranea Schultze \& Müller, 1857 & Mogi das Cruzes, Avaré, São Paulo \\
\hline 244 & & Geoplana braunsi von Graff, 1899 & Amparo, Santos, Mogi das Cruzes \\
\hline 245 & & Geoplana burmeisteri Schultze \& Müller, 1857 & Ubatuba, São Paulo \\
\hline 246 & & Geoplana caapora Froehlich, 1956 & Apiaí \\
\hline 247 & & Geoplana cafusa Froehloch, 1956 & Ubatuba \\
\hline 248 & & Geoplana carinata Riester, 1938 & $\begin{array}{l}\text { Ribeirão Pires, São Paulo, Santos, } \\
\text { Pirassununga, Itapecerica, Juquiá, } \\
\text { Mongaguá, Mogi das Cruzes }\end{array}$ \\
\hline 249 & & Geoplana carrierei von Graff, 1897 & São Paulo, Santo André \\
\hline 250 & & Geoplana chita Froehlich, 1956 & Eldorado \\
\hline 251 & & Geoplana chiuna E.M. Froehlich, 1955 & Pirassununga \\
\hline 252 & & Geoplana crioula E. M. Froehlich, 1955 & São Paulo \\
\hline 253 & & Geoplana divae Marcus, 1951 & Santo André, Santos \\
\hline 254 & & Geoplana evelinae Marcus, 1951 & Mongaguá, Santo André \\
\hline 255 & & Geoplana ferussaci von Graff, 1899 & São Paulo \\
\hline 256 & & Geoplana hina Marcus, 1951 & Santo André \\
\hline 257 & & Geoplana itatiayana Schirch, 1929 & São Paulo \\
\hline 258 & & Geoplana livia E. M. Froehlich, 1955 & São Paulo \\
\hline 259 & & Geoplana metzi von Graff, 1899 & São Paulo, Ribeirão Pires \\
\hline 260 & & Geoplana multicolor von Graff, 1899 & São Paulo \\
\hline 261 & & Geoplana pavani Marcus, 1951 & Mogi das Cruzes \\
\hline 262 & & Geoplana phocaica Marcus, 1951 & São Paulo \\
\hline 263 & & Geoplana picta Froehlich, 1956 & Ubatuba \\
\hline 264 & & Geoplana poca Froehlich, 1958 & Itanhaém \\
\hline 265 & & Geoplana preta Riester, 1938 & São Paulo \\
\hline 266 & & Geoplana quagga Marcus, 1951 & São Paulo \\
\hline 267 & & Geoplana regia E.M. Froehlich, 1955 & Araraquara \\
\hline 268 & & Geoplana schubarti Froehlich, 1958 & Itanhaém, São Paulo, \\
\hline 269 & & Geoplana toriba Froehlich, 1958 & Juquiá \\
\hline 270 & & Geoplana tuxaua E.M. Froehlich, 1955 & São Paulo \\
\hline 271 & & Geoplana vaginuloides (Darwin, 1844) & São Paulo, Itanhaém, Eldorado \\
\hline 272 & & Giradia arndti (Marcus, 1946) & Campos do Jordão \\
\hline 273 & & Girardia chilla (Marcus, 1954) & Salesópolis \\
\hline 274 & & Girardia hypoglauca (Marcus, 1948) & São Paulo \\
\hline 275 & & Girardia nonatoi (Marcus, 1946 ) & São Paulo \\
\hline 276 & & Girardia schubarti (Marcus, 1946) & $\begin{array}{l}\text { Campos do Jordão, Salesópolis, Botucatu, } \\
\text { São Paulo, Monte Alegre, São Carlos }\end{array}$ \\
\hline 277 & & Girardia tigrina (Girard, 1850) & São Carlos, Botucatu, São Paulo \\
\hline 278 & & Issoca jandaia Froehlich, 1955 & São Paulo \\
\hline 279 & & Issoca potyra Froehlich, 1958 & Eldorado \\
\hline 280 & & Issoca rezendei (Schirch, 1929) & Ubatuba, São Paulo, Itanhaém \\
\hline 281 & & Leucolesma corderoi Marcus, 1948 & Santos \\
\hline 282 & & Nerpa evelinae Marcus, 1948 & Guarujá, Santos \\
\hline 283 & & Notogynaphallia caissara (E.M. Froehlich, 1955) & Ubatuba, Ribeirão Pires, Itanhaém \\
\hline 284 & & Notogynaphallia ernesti Leal-Zanchet \& E.M. Froehlich, 2006 & $\begin{array}{l}\text { Pirassununga, Valinhos, Jundiaí, São Paulo, } \\
\text { Mogi das Cruzes, Ibiúna, Ribeirão Pires }\end{array}$ \\
\hline 285 & & Notogynaphallia goetschi (Riester, 1938) & $\begin{array}{l}\text { Ubatuba, São Paulo, Mogi das Cruzes, Santo } \\
\text { André, Santos, Guapiara, Mongaguá, Eldorado }\end{array}$ \\
\hline 286 & & Notogynaphallia parca (E.M. Froehlich, 1955) & São Paulo \\
\hline 287 & Tricladida & Notogynaphallia plumbea (Froehlich, 1956) & São Paulo \\
\hline 288 & & Notogynaphallia sexstriata ( von Graff, 1899) & São Paulo \\
\hline
\end{tabular}


Tabela 1. Continuação...

\begin{tabular}{|c|c|c|c|}
\hline $\mathbf{N}^{\circ}$. & Táxon & Espécie descritor & $\begin{array}{c}\text { Ocorrência } \\
{[\text { [em município(s) do estado SP] }}\end{array}$ \\
\hline 289 & & Pasipha astraea (Marcus, 1951) & São Paulo, Mogi das Cruzes \\
\hline 290 & & Pasipha chimbeva (E.M. Froehlich, 1955) & São Paulo \\
\hline 291 & & Pasipha pasipha (Marcus, 1951) & São Paulo \\
\hline 292 & & Pasipha pinima (E.M. Froehlich, 1955) & São Paulo \\
\hline 293 & & Pasipha rosea (E.M. Froehlich, 1955) & Pirassununga, São Paulo, São Simão \\
\hline 294 & & Pasipha tapetilla (Marcus, 1951) & Pirassununga, Ubatuba \\
\hline 295 & & Pasipha trina (Marcus, 1951) & Santo André \\
\hline 296 & & Pseudogeoplana brasiliensis (Blainville, 1826) & Santos \\
\hline 297 & & Puiteca camica du Bois-Reymond-Marcus, 1953 & Ubatuba \\
\hline 298 & & Rhodax evelinae Marcus, 1946 & São Paulo \\
\hline 299 & & Rhynchodemus piptus Marcus, 1952 & São Paulo \\
\hline 300 & & Rhynchodemus schubarti du Bois-Reymond Marcus, 1955 & Pirassununga \\
\hline 301 & & Rhynchodemus scius du Bois-Reymond Marcus, 1955 & Ubatuba, Pirassununga \\
\hline 302 & & Tiddles evelinae Marcus, 1963 & Cananéia \\
\hline 303 & & Vatapa gabriellae Marcus, 1948 & Santos \\
\hline 304 & Typhloplanoida & Bothromesostoma evelinae Marcus, 1946 & São Paulo \\
\hline 305 & & Mesostoma ehrenbergi (Focke, 1836) & São Paulo \\
\hline 306 & & Olisthanella opistomiformis Nasonov, 1924 & São Paulo \\
\hline 307 & & Phaenocora bresslaui Marcus, 1946 & São Paulo \\
\hline 308 & & Phaenocora chloroxanta Mracus, 1946 & São Paulo \\
\hline 309 & & Phaenocora evelinae Marcus, 1946 & São Paulo \\
\hline 310 & & Strongylostoma dicorhymbum Marcus, 1946 & São Paulo \\
\hline
\end{tabular}

O conhecimento sobre a biologia e ecologia das espécies é muito pequeno. As relações filogenéticas entre os táxons não foi ainda estudada. O principal impedimento para ter uma ideia básica da diversidade e biologia das espécies, bem como da evolução dos grupos, é a falta de especialistas. O atual quadro de taxonomistas não dará conta desta enorme tarefa sem a incorporação de novos jovens cientistas.

\section{Agradecimentos}

Ao Dr. Carlos Lamas (MZUSP), Ana Vasques (MZUSP), Dra. Suzana B. Amato (UFRGS) pelo fornecimento de informações sobre as coleções; ao Dr. Marcos R. Hara (EACH/USP) pela confecção da figura; a Jim Hesson pela revisão do inglês. FC tem apoio da FAPESP.

\section{Referências Bibliográficas}

CANNON, L. R. G., 1996. Turbellaria of the World. A guide to families and genera. Queensland Museum, Brisbane. 136 pp.

CARBAYO, F. \& FROEHLICH, E.M. 2008. Estado do conhecimento dos macroturbelários (Platyhelminthes) do Brasil. Biota Neotrop. 8(4):177-197.

CARBAYO, F., LEAL-ZANCHET, A.M. \& VIEIRA, E.M. 2002. Terrestrial flatworm (Platyhelminthes: Tricladida: Terricola) diversity $v s$. maninduced disturbance in a subtropical rainforest from Southern Brazil. Biodiversity Conserv. 11:1091-1104.

CARBAYO, F., LEAL-ZANCHET, A. M., FROEHLICH, E. M. \& AMATO, S. B. 2009. Turbelários (Platyhelminthes). In Estado da arte e perspectivas para a zoologia no Brasil (R. Rocha \& W. Boeger, org.). Ed. UFPR, Curitiba, p.49-64.
FORNERIS, L. 1999. Platelmintos turbelários. In Biodiversidade do Estado de São Paulo, Brasil: síntese do conhecimento ao final do século XX. Invertebrados de Água Doce (C.A. Joly \& C.E.M. Bicudo, org.). FAPESP, São Paulo, p.19-23.

HOOGE, M.D. \& ROCHA, C.E.F. 2006. Acoela (Acoelomorpha) from the northern beaches of the state of São Paulo, Brazil, and a systematic revision of the family Otocelididae. Zootaxa 1335:1-50.

JOLY, C.A., BICUDO, C.E.M.M. 1999. orgs. Biodiversidade do Estado de São Paulo, Brasil:síntese do conhecimento ao final do Século XX. 3: Invertebrados Marinhos. FAPESP, São Paulo.

RODRIGUES, S.D.A. \& FROEHLICH, E.M. 1998. Filo Platyhelminthes Classe Turbellaria. In Biodiversidade do Estado de São Paulo, Brasil: síntese do conhecimento ao final do século XX. Invertebrados hos (C.A. Joly \& C.E.M. Bicudo, org.). FAPESP, São Paulo, v.3.

RIEGER, R.M.S., TYLER, J.P.S., SMITH III \& RIEGER, G.E. 1991. Platyhelminthes: Turbellaria. In F.E.Harrison \& B.J. Bogitsh (eds), Microscopic Anatomy of Invertebrates, Vol.3, Platyhelminthes and Nemertinea. Wiley Liss: 7-140.

SCHOCKAERT, E.R., HOOGE, M., SLUYS, R., SCHILLING, S., TYLER, S. \& ARTOIS, T. 2008. Global diversity of free living flatworms (Platyhelminthes, "Turbellaria") in freshwater. Hydrobiologia 595:41-48.

SLUYS, R. 1999. Global diversity of land planarians (Platyhelminthes, Tricladida, Terricola): a new indicator-taxon in biodiversity and conservation studies. Biodiversity Conserv. 8(12):1663-1681.

TYLER, S., SCHILLING, S., HOOGE, M. \& BUSH, L.F. (comp.). 2006-2009. Turbellarian taxonomic database. Version $1.5 \mathrm{http}: / /$ turbellaria.umaine. edu (último acesso em 11/05/2010). 


\section{Apêndice 1}

Apêndice 1. Referências complementares.

Appendix 1. Complementary references.

ALMEIDA, E.J.C.D., YAMADA, C.M. \& FROEHLICH, E.M. 1991. Cytogenetic studies of two land planarian species from Brazil: Geoplana marginata and Issoca rezendei (Tricladida, Terricola). Hydrobiologia 227:169-173. http://dx.doi.org/10.1007/BF00027598

ALMEIDA, E.C.J.D., YAMADA, C.M. \& FROEHLICH, E.M. 1988. Estudos cromossômicos em duas espécies de planárias terrestres: Geoplana abundans Graff e Issoca rezendei (Schirch) (Platyhelminthes, Tricladida, Terricola). Ciênc. Cult. Supl. 40:764-765.

ÁLVAREZ, L. 1996. Contribuição a biologia e a cariologia de sete espécies de planárias terrestres pertencentes as famílias Geoplanidae e Rhynchodemidae. Dissertação de mestrado, Universidade de São Paulo, São Paulo.

ÁLVAREZ, L. 2001. Análises citogenéticas em planárias terrestres pertencentes às famílias Geoplanidae e Rhynchodemidae. Tese de doutorado, Universidade de São Paulo, São Paulo.

ÁLVAREZ, L. \& ALMEIDA, E.J.C.D. 1999. Comparative karyotypic analysis of two land planarian species from Brazil: Geoplana burmeisteri and Geoplana carinata (Tricladida, Terricola). Hereditas 131(1):1-4. http://dx.doi.org/10.1111/j.1601-5223.1999.t01-1-00001.x

ÁLVAREZ, L. \& ALMEIDA, E.J.C.D. 2002. The first record of polyploidy in a land planarian species (Geoplana burmeisteri). Caryologia 55(3):235-239.

ÁLVAREZ, L. \& ALMEIDA, E.J.C.D. 2007. Comparative karyotype analysis in diploid and triploid Dolichoplana carvalhoi (Tricladida, Terricola, Rhynchodemidae) from Brazil. Genet. Mol. Biol. 30(2):375-379.

ANTONIAZZI, M.M. 1989. Estudo ultra-estrutural comparativo do trato digestivo de Macrostomum gigas, Stenostomum grande e Prorhynchus stagnalis (Platyhelminthes; Turbellaria). Dissertação de mestrado, Universidade de São Paulo, São Paulo.

ANTONIAZZI, M.M. 1997. Estudo por microscopia eletrônica de transmissão e de varredura do microturbelário de água doce Stenostomum grande (Plathelminthes, Catenulida), com vistas ao processo de paratomia. Tese de doutorado, Universidade de São Paulo, São Paulo.

BLAINVILLE, D.D. 1826. Planaires. In Dictionnaire Des Sciences Naturelles, 61: 215-216. Paris.

CARBAYO, F. 2006. Redescription of two land planarian species of Notogynaphallia Ogren \&. Kawakatsu (Platyhelminthes, Tricladida, Geoplaninae) and confirmation of the heterogeneity of the genus. Braz. J. Zool. 23(3):746-757. http://dx.doi.org/10.1590/S0101-81752006000300020

CARBAYO, F. \& LEAL-ZANCHET, A.M. 2003. Two new genera of Geoplaninae (Terricola: Tricladida: Platyhelminthes) of Brazil in the light of cephalic apomorphies. Invert. Syst. 17(3):449-468. http://dx.doi.org/10.1071/IT01035

CARBAYO, F., PEDRONI, J. \& FROEHLICH, E.M. 2008. Colonization and extinction of land planarians (Platyhelminthes, Tricladida) in a Brazilian Atlantic Forest regrowth remnant. Biol Invasions 10(7):1131-1134. http://dx.doi.org/10.1007/s10530-007-9190-1

CARLÉ, R. 1935. Beiträge zur Embriologie der Landplanarien. Ztschr. Morphol. Ökol. Tiere 29(4):527-558.

CARVALHO, I.M.D., BRUNNER JUNIOR, A. \& VALENTE, M.M. 1984a. Relationship between the dorsoventral muscle fibers and the epidermis of Geoplana carinata Riester, 1938 (Platyhelminthes, Turbellaria, Tricladia). Zool. Jb. Anat. 111:25-33.

CARVALHO, I.M.D., VALENTE, M.M. \& KÖHLER, F.W. 1984b. Die Feinstruktur der Basalmembran bei Geoplana carinata Riester, 1938 (Platyhelminthes - Turbellaria). Z. Mikrosk. - Anat. 98(3):403-416.

CORRÊA, D.D. 1947. A primeira Dolichoplana (Tricladida, Terricola) do Brasil. Bol. Fac. Fil. Ci. Letras, Univ. São Paulo, Zool. 12:57-81.

CORRÊA, D.D. 1949. Sobre o gênero Zygantroplana. Bol. Fac. Fil. Ciênc., Sér. Zool. 99:173-218.

CORRÊA, D.D. 1958. A new polyclad from Brazil. Bol. Inst. Oceanogr. São Paulo 7:81-86.

CORRÊA, D.D. 1964. Turbellaria hos. In História natural de organismos aquáticos do Brasil. Fapesp, São Paulo, p.101-102.

DIESING, K.M. 1861. Revision der Turbellarien. Abtheilung: Dendrocoelen. Sitzungsberichte d. mathemat.-Naturwiss. Classe der kaiserl. Akad. Wiss. 44 I Abt. 6-10:485-578.

DOLCI, I.A. 1978a. Estudo sobre o comportamento de planárias em situação padronizada. Uma contribuição à categorização e descrição do comportamento de Dugesia tigrina. Dissertação de mestrado, Universidade de São Paulo, São Paulo.

DOLCI, I.A. 1978b. A planária no ambiente de manutenção: um estudo biológico e psicológico. Tese de doutorado, Universidade de São Paulo, São Paulo. DU BOIS-REYMOND MARCUS, E. 1951a. Contributions to the natural history of Brazilian Turbellaria. Comun. Zool. Mus. Hist. Nat. Montevideo 3(63):1-25. DU BOIS-REYMOND MARCUS, E. 1951b. On South American geoplanids. Bol. Fac. Fil. Ci. Letras, Univ. São Paulo, Zool. 16:217-255.

DU BOIS-REYMOND MARCUS, E. 1955a. Chave dos Polycladida do litoral de São Paulo. Bol. Fac. Fil. Ci. Letras, Univ. São Paulo, Zool. 19:281-288.

DU BOIS-REYMOND MARCUS, E. 1955b. On Turbellaria and Polygordius from the Brazilian coast. Bol. Fac. Fil. Ci. Letras, Univ. São Paulo, Zool. $20: 19-53$.

DU BOIS-REYMOND MARCUS, E. 1957. On Turbellaria. Acad. Brasil. Ciênc. 29(1):153-191.

DU BOIS-REYMOND MARCUS, E. 1958. On South American Turbellaria. Acad. Brasil. Ciênc. 30(3):391-417.

DU BOIS-REYMOND MARCUS, E. 1965. Drei neotropische Turbellarien. Sitz. - Ber. Ges. naturf. Freunde, Berlin 5:129-135.

DU BOIS-REYMOND MARCUS, E. \& MARCUS, E. 1968. Polycladida from Curação and faunistically related regions. Stud. Fauna Curação 26:1-106.

FALLENI, A., LUCCHESI, P., GHEZZANI, C., SILVEIRA, M. \& GREMIGNI. V. 2006. Ultrastructural and cytochemical aspects of the female gonad of Geoplana burmeisteri (Platyhelminthes, Tricladida, Terricola). Jour. Morphol. 267:318-332. PMid:16323219. http://dx.doi.org/10.1002/jmor.10405

FAUBEL, A. 1983. The Polycladida, Turbellaria. Proposal and establishment of a new system. Part I. The Acotylea. Mitt. Hamb. Zool. Mus. Inst. 80:17-121.

FAUBEL, A. 1984. The Polycladida, Turbellaria. Proposal and establishment of a new system. Part II. The Cotylea. Mitt. Hamb. Zool. Mus. Inst. 80:189-259.

FERNANDES, M.C. 2000. O sistema nervoso da região cefálica da planária terrestre "Bipalium kewense". Tese de doutorado, Universidade de São Paulo,

São Paulo.

FÉRUSSAC, A.E.D. 1821. Note sur une nouvelle Espèce de ver terrestre du Brésil. Journ. de Physique 92:233-235.

FROEHLICH, C.G. 1954. Sobre morfologia e taxonomia das Geoplanidae. Tese de doutorado, Universidade de São Paulo. 
Catálogo dos “Turbellaria” (Platyhelminthes) do Estado de São Paulo

FROEHLICH, C.G. 1955a. Sôbre morfologia e taxonomia das Geoplanidae. Bol. Fac. Fil. Ci. Letras, Univ. São Paulo, Zool. 19:195-279.

FROEHLICH, C.G. 1955b. Notas sôbre Geoplanas brasileiras. Pap. Avul. Dep. Zool. 12(7):189-198.

FROEHLICH, C.G. 1955c. On the biology of land planarians. Bol. Fac. Fil. Ci. Letras, Univ. São Paulo, Zool. 20:263-271.

FROEHLICH, C.G. 1956. Tricladida Terricola das regiões de Teresópolis e Ubatuba. Pap. Avul. Dep. Zool. 12:313-344.

FROEHLICH, C.G. 1958. On a collection of brazilian land planarians. Bol. Fac. Fil. Ci. Letras, Univ. São Paulo, Zool. 21:93-121.

FROEHLICH, C.G. 1959. On geoplanids from Brazil. Bol. Fac. Fil. Ci. Letras, Univ. São Paulo, Zool. 22:201-265.

FROEHLICH, C.G. 1959. Planárias terrestres do sul do Brasil. Tese de livre docência, Universidade de São Paulo.

FROEHLICH, C.G. 1964. Turbellaria límnicos. In História natural de organismos aquáticos do Brasil. Fapesp, São Paulo, p.99-100.

FROEHLICH, C.G. 1966. Notas sobre a ecologia de planárias terrestres. In Progresos en biologia del suelo. Montevideo, Uruguay, $299-301$.

FROEHLICH, C.G. 1967. A contribution to the zoogeography of neotropical land planarians. Acta Zool. Lilloana 23:153-162.

FROEHLICH, E.M. 1954. Sobre espécies brasileiras do gênero Geoplana. Tese de doutorado, Universidade de São Paulo.

FROEHLICH, E.M. 1955a. Sôbre espécies brasileiras do gênero Geoplana. Bol. Fac. Fil. Ci. Letras, Univ. São Paulo, Zool. 19:289-369.

FROEHLICH, E.M. 1955b. Chave para a classificação das Geoplanas brasileiras. Pap. Av. Dep. Zool. 12(8):201-214.

FROEHLICH, E.M. \& LEAL-ZANCHET, A.M. 2003. A new species of terrestrial planarian of the genus Notogynaphallia (Platyhelminthes, Tricladida, Terricola) from south Brazil and some comments on the genus. Rev. Bras. Zool. 20(4):745-753. http://dx.doi.org/10.1590/S0101-81752003000400030

HYMAN, L.H. 1955. Miscellaneous e and terrestrial flatworms from South America. Am. Mus. Novitates 1742:1-33.

JORGE, M.A.P. 2003. Estudos sobre a biologia e a citogenética em planárias de água doce da família Dugesiidae. Dissertação de mestrado, Universidade de São Paulo, São Paulo.

KAWAKATSU, M., HAUSER, J. \& FRIEDRICH, S.M.G. 1983B. Morphological, karyological and taxonomic studies of freshwater planarians from South Brazil. V. Dugesia tigrina (Girard, 1850) from município Botucatu, Estado de São Paulo, and Dugesia schurbati (Marcus, 1946) from the vicinity of São Paulo. Bull. Fuji Women's Coll. 21:147-163.

KENK, R. 1974. Index to the genera and species of the freshwater triclads (Turbellaria) of the world. Smithsonian Contr. Zool. 183:1-90. http://dx.doi. org/10.5479/si.00810282.183

LEAL-ZANCHET, A.M. \& FROEHLICH, E.M. 2001. A species complex in the genus Notogynaphallia (Tricladida: Terricola). Belg. J. Zool. 131 (supl.):225-226.

LEAL-ZANCHET, A.M. \& FROEHLICH, E.M. 2006. A species complex in the genus Notogynaphallia Ogren and Kawakatsu (Platyhelminthes: Tricladida: Terricola) with a taxonomic revision of homonyms of Geoplana marginata Schultze \& Müller and a reinterpretation of Notogynaphallia caissara (Froehlich) anatomy. Belg. J. Zool. 136(1):81-100.

LEAL-ZANCHET, A.M. \& SOUZA, S.A.D. 2003. Redescrição de Choeradoplana iheringi Graff (Platyhelminthes, Tricladida, Terricola). Rev. Bras. Zool. 20(3):523-530. http://dx.doi.org/10.1590/S0101-81752003000300026

MARCUS, E. 1943. O Turbelaria Mesostoma ehrenbergii Focke 1836 no Brasil. Bol. Industria Animal N. S. 6:12-15.

MARCUS, E. 1945. Sobre microturbellarios do Brasil. Comunicaciones Zoológicas del Museo de Historia Natural Montevideo 1(25):1-74.

MARCUS, E. 1946. Sobre Turbellaria brasileiros. Bol. Fac. Fil. Ci. Letras, Univ. São Paulo, Zool. 11:5-253.

MARCUS, E. 1947. Turbelários hos do Brasil. Bol. Fac. Fil. Ci. Letras, Univ. São Paulo, Zool. 12:99-215.

MARCUS, E. 1948. Turbellaria do Brasil. Bol. Fac. Fil. Ci. Letras, Univ. São Paulo, Zool. 13:113-243.

MARCUS, E. 1949. Turbellaria brasileiros (7). Bol. Fac. Fil. Ci. Letras, Univ. São Paulo, Zool. 14:7-155.

MARCUS, E. 1950. Turbellaria brasileiros (8). Bol. Fac. Fil. Ci. Letras, Univ. São Paulo, Zool. 15:5-191.

MARCUS, E. 1951. Turbellaria brasileiros (9). Bol. Fac. Fil. Ci. Letras, Univ. São Paulo, Zool. 16:5-215.

MARCUS, E. 1952. Turbellaria brasileiros (10). Bol. Fac. Fil. Ci. Letras, Univ. São Paulo, Zool. 17:5-187.

MARCUS, E. 1954. Turbellaria Brasileiros - XI. Pap. Avulsos Dep. Zool. Secr. Agric. 24:419-489.

MARCUS, E. 1963. Eine neue Meerestriklade von Sao Paulo. Zool. Beitr. (N.S.) 9:441-446.

MARTINS, M.E.Q.P. 1970. Two new species of Dugesia (Tricladida Paludicola) from the State of São Paulo, Brazil. An. Acad. Brasil. Ciênc. 42:113-118.

MELO, A.S. \& ANDRADE, C.F. 2001. Differential predation of the planarian Dugesia tigrina on two mosquito species under laboratory conditions. J. Am. Mosquito Control Ass. 17(1):81-83.

MELO, A.S., MACEDO, C.C. \& ANDRADE, C.F.S.D. 1995. Eficiência de Dugesia tigrina (Girard) Turbellaria, Tricladida) como agente controlador de imaturos do mosquito Aedes albopictus (Skuse) em pneus armadilha. An. Soc. Entomol. Bras. 25(2):321-327.

MIGOTTO, A.E. \& MARQUES, A.C. 2006. Invertebrados hos. In Avaliação do estado do conhecimento biodiversidade brasileira (T. Lewinsohn, org.). Brasília: Ministério do Meio Ambiente, v.1, p.149-202.

MOLINA, F.M.R.L. 1976. Reprodução e ciclo biológico de Dugesia tigrina (Girard 1850) (Turbellaria, Tricladida, Paludicola). Dissertação de mestrado, Universidade de São Paulo.

NOREN, M. \& JONDELIUS, U. 2002. The phylogenetic position of the Prolecithophora (Rhabditophora, 'Platyhelminthes'). Zool. Scr. 31(4):403-414. http:// dx.doi.org/10.1046/j.1463-6409.2002.00082.x

OGREN, R.E \& KAWAKATSU, M. 1998. American neartic and neotropical land planarian (Tricladida: Terricola) faunas. Pedobiologia 42:441-451.

OGREN, R.E. \& KAWAKATSU, M. 1990. Index to the species of the family Geoplanidae (Turbellaria, Tricladida, Terricola) Part I: Geoplaninae. Bull. Fuji Women's College 28(I):79-166.

OGREN, R.E., KAWAKATSU, M. \& FROEHLICH, E.M. 1997. Additions and corrections of the previous land planarian indices of the world (Turbellaria, Seriata, Tricladida, Terricola) Addendum IV. Geographic locus index: Bipaliidae, Rhynchodemidae (Rhynchodeminae, Microplaninae), Geoplanidae (Geoplaninae, Caenoplaninae, Pelmatoplaninae). Bull. Fuji Womens’College 35(I):63-103.

OPHELIS DE ALMEIDA, F.J. 1992. Morfologia de Pasipha pasipha (Marcus, 1951) (Platyhelminthes: Tricladida: Terricola). Dissertação de mestrado, Universidade de São Paulo, São Paulo. 
PRUDHOE, S. 1985. A monograph on Polyclad Turbellaria. Oxford University Press, London.

RAMOS, M.C. 1993. Aspectos da biologia de Issoca rezendei (Schirch, 1929) (Tricladida: Terricola: Geoplanidae). Dissertação de mestrado, Universidade de São Paulo, São Paulo.

RIESTER, A. 1938. Beiträge zur Geoplaniden-Fauna Brasiliens. Abhandl. senkenberg. naturf. Ges. 441:1-88.

RODRIGUES, R.M.A. 1972. Ciclo biológico de Geoplana burmeisteri Max Schultze, 1857 (Turbellaria, Tricladida, Terricola). Dissertação de mestrado, Universidade de São Paulo, São Paulo.

SAFADI, R.S. 1993. Emprego de planárias de água doce, Girardia tigrina (Girard, 1850) (Platyhelminthes, Tricladida, Paludicola) na valiação de compostos metálicos: proposta metodológica. Dissertação de mestrado, Universidade de São Paulo, São Paulo.

SAWAYA, P. \& UNGARETI, M.D. 1948. Influência da temperatura sobre o consumo de oxigênio pelas planárias. Bol. Fac. Fil. Ci. Letras, Univ. São Paulo, Zool. 13:329-334.

SILVEIRA, M. 1967. Ultraestruturas ciliares de Turbelarios e suas implicações fisiológicas. Tese de doutorado. Universidade de São Paulo, São Paulo.

SILVEIRA, M. \& CORINNA, A. 1976. Fine structural observations on the protonephridium of the terrestrial triclad Geoplana pasipha. Cell. Tiss. Res. 168:455-463. PMid:1277279. http://dx.doi.org/10.1007/BF00215996

SLUYS, R. 1989. A Monograph of the e Triclads. A. A. Balkema, Rotterdam.

SLUYS, R. 1990. A monograph of the Dimarcusidae (Platyhelminthes, Seriata, Tricladida). Zool. Scr. 19(1):13-29. http://dx.doi.org/10.1111/j.1463-6409.1990. tb00237.x

SLUYS, R. \& KAWAKATSU, M. 2005. Biodiversity of e planarians revisited (Platyhelminthes, Tricladida, Maricola). J. Nat. Hist. 39(6):445-467.

SLUYS, R. 1996. Reconsiderations of species status of some South American planarians (Platyhelminthes: Tricladida: Paludicola). Proc. Biol. Soc. Washington 109(2):229-235. http://dx.doi.org/10.1080/00222930410001671309

SMITH, E.H. 1960. On a new Polyclad commensal of Prosobranchs. An. Acad. Brasi. Ci. 32(3-4):1-4.

SOUZA, S.A.D. \& LEAL-ZANCHET, A.M. 2004. Histological and histochemical characterization of the secretory cells of Choeradoplana iheringi Graff, 1899 (Platyhelminthes: Tricladida: Terricola). Braz. J. Biol. 64(3):511-522.

VON GRAFF, L.V. 1899. Monographie der Turbellarien: II. Tricladida Terricola. Engelmann, Leipzig. 